\title{
The Mathematics of Medical Imaging: A Beginner's Guide
}

\author{
T.G. Feeman
}

New York, NY: Springer, 2010, 141 pages, $\$ 49.95$

This is a mathematics book. It is not a "math for the math-averse" book, and it is not a numerical methods book. It is a textbook that presents a compact, rigorous treatment of basic tomographic image reconstruction at a level suitable for an undergraduate who is strong in math. The chapters address x-rays, the Radon transform, backprojection, complex numbers, the Fourier transform, the Central Slice Theorem, filtered backprojection and the Hilbert transform, filters and convolution, sampled data and discrete image reconstruction, algebraic reconstruction techniques, and MRI. Appendices provide background information on integrability and a list of topics for further study. The text deals primarily with a single-slice, parallel-beam geometry. It discusses the Radon transform and filtered backprojection in great detail. It also has a nice section on algebraic reconstruction techniques, as they are illustrative of interesting mathematics, even if not widely used in modern practice.

This book is valuable, for it addresses with care and rigor the relevance of a variety of mathematical topics to a real-world problem. This book is written for readers with mathematical talent, not for poseurs like me who struggled in math majors' math classes. I intend to add this book to the list of supplemental readings for my medical physics students, as it should reduce the frustration of my mathematically sophisticated students who could benefit from this text's careful treatment of topics that I present only verbally, conceptually, and with great waving of hands. There are certainly more lengthy and detailed books on the topic, such as those by Natterer and Epstein (1-3) that are cited in the book's bibliography, but this book is just right in length and depth for its primary audience-mathematically adroit beginners. Mathematicians could then graduate to Natterers' books while physicists and engineers might progress to Epstein's and Herman's $(3,4)$.

I have only a few quibbles with the book. One minor point is that the reader is led to infer that all random processes are zeromean. Another is some statements made in passing that imply that helical scanning and cone beam geometry in CT inherently reduce the patient dose by faster data acquisition as if the patient dose were solely a consequence of the beam-on time. The author repeats the common assertion that twice (rather than strictly greater than twice) the maximum frequency of a band-limited signal is an adequate uniform sampling rate.

The book says little about noise and statistics and even less about the treatment of nonideal conditions such as beam-hardening effects in CT and attenuation, scatter, and spatially variable resolution effects in SPECT and PET. Other than Kaczmarz's method for algebraic reconstruction, iterative reconstruction is not developed. These absences are hardly defects, given the introductory purpose of the book and its brevity.

Presenting MRI in a single chapter is a daunting task, and the chapter on MRI works all the way up to 1-dimensional frequency encoding only to end abruptly. Within a few more pages, the view of the raw data from 2-dimensional MRI as the Fourier transform of the image plane could have been developed and then tied through the Central Slice Theorem to reconstruction in PET, SPECT, and CT, thereby connecting the modalities and unifying the topics of the book.

This book is well written. It serves its purpose of focusing a variety of mathematical topics onto a real-world application that is in its essence mathematics. The course from which it sprang sounds like a lot more fun than any math course that I ever took.

\section{REFERENCES}

1. Natterer F. The Mathematics of Computerized Tomography. Philadelphia, PA: Society for Industrial Applications of Mathematics; 2001.

2. Natterer F, Wuebbeling F. Mathematical Methods in Image Reconstruction. Philadelphia, PA: Society for Industrial Applications of Mathematics; 2001.

3. Epstein CL. Introduction to the Mathematics of Medical Imaging. 2nd ed. Philadelphia, PA: Society for Industrial Applications of Mathematics; 2008.

4. Herman GT. Fundamentals of Computerized Tomography: Image Reconstruction from Projections. 2nd ed. Dordrecht, The Netherlands: Springer; 2009.

\section{Richard Wendt III}

The University of Texas

M.D. Anderson Cancer Center

1515 Holcombe Blvd., Unit 1352

Houston, TX 77030

E-mail: rwendt@mdanderson.org 